\title{
Fermi-edge singularities in linear and nonlinear ultrafast spectroscopy
}

\author{
D. Porras, J. Fernández-Rossier,* and C. Tejedor \\ Departamento de Física Teórica de la Materia Condensada, Universidad Autónoma de Madrid, Cantoblanco, 28049 Madrid, Spain
}

(Received 5 September 2000; revised manuscript received 30 January 2001; published 6 June 2001)

\begin{abstract}
We discuss Fermi-edge singularity effects on the linear and nonlinear transient response of an electron gas in a doped semiconductor. We use a bosonization scheme to describe the low-energy excitations, which allows us to compute the time and temperature dependence of the response functions. Coherent control of the energy absorption at resonance is analyzed in the linear regime. It is shown that a phase shift appears in the coherent control oscillations, which is not present in the excitonic case. The nonlinear response is calculated analytically and used to predict that four wave-mixing experiments would present a Fermi-edge singularity when the exciting energy is varied. A new dephasing mechanism is predicted in doped samples that depends linearly on temperature and is produced by the low-energy bosonic excitations in the conduction band.
\end{abstract}

DOI: 10.1103/PhysRevB.63.245321

PACS number(s): 78.47.+p

\section{INTRODUCTION}

The promotion of an electron from a localized state in the valence band to an empty state in a partially filled conduction band is accompanied by a dynamical response of the Fermi gas. The enhancement of the absorption probability when the new electron is promoted just above the Fermi level is known as the Fermi-edge singularity (FES). ${ }^{1}$ This phenomenon has been observed in continuous-wave spectroscopy in a variety of doped semiconductor heterostructures. $^{2,3}$ FES arises as a result of the interplay between two different physical processes: the sudden appearance of a hole potential and the presence of an extra electron at the conduction band. Both effects produce charge-density oscillations involving low-energy electron-hole pairs. The constructive interference between these two effects gives the FES. Following the seminal work of Schotte and Schotte, ${ }^{4}$ these low-energy electron-hole pairs can be described as Tomonaga bosons.

Coherent ultrafast spectroscopy of undoped semiconductors, where excitons are the relevant excitation, has been much more widely addressed than that of the doped case. Both linear and nonlinear techniques, like coherent control (CC) and four wave mixing (FWM), have been used to study the decay of the optical coherence induced by the laser in undoped samples. ${ }^{5}$ In the case of doped systems, only a few experiments has been performed. Kim et al. ${ }^{6}$ carried out FWM experiments in n-doped GaAs quamtum wells that presented FES in continuous-wave spectroscopy. In this experiment it was determined that the carrier-carrier scattering rate was a decreasing function of the exciting energy (above the Fermi energy), in agreement with Landau theory. However, the spectral width of their laser pulses was larger than the Fermi-energy of the electron gas so that Fermi-edge excitations coexist with higher-energy electrons. Bar-Ad et al. performed FWM experiments under strong magnetic fields finding indications of the nonlinear response of the FES. ${ }^{7}$ Brener et $\mathrm{al}^{8}{ }^{8}$ performed off-resonant pump and probe experiments in $n$-doped GaAs QW, probing the ac Stark shift (a nonresonant nonlinearity) in contrast with the works by Kim et al., and Bar-ad et al. which measured resonant nonlinearities. From the theory side, Perakis et al. ${ }^{9-11}$ have studied the coherent nonlinear response of the FES, either under ultrashort laser pulses, or under nonresonant excitation, i.e., when the nonlinearity comes from an intense laser pulse spectrally peaked below the absorption threshold.

Our paper addresses a physical situation slightly different from all of the above; a doped semiconductor, in zero magnetic field, is excited by laser pulses spectrally peaked around the absorption threshold, so that absorption takes place. Moreover, the laser pulses are spectrally narrow (compared to the Fermi energy $\epsilon_{F}$ measured from the bottom of the conduction band) so that the photoexcited electrons have energies close to the Fermi level, but the pulses are shorter than $T_{2}$ so that transient coherent effects can be observed. ${ }^{5}$

Our main findings are (i) CC of the energy absorbed by the system (the analogous of $\mathrm{CC}$ of the exciton density ${ }^{12-14}$ ) can be performed in doped samples. CC oscillations show a characteristic phase shift, which depends on the exponent of the continuous wave FES. (ii) The intensity of the FWM signal shows a singularity when the exciting frequency is varied near the Fermi edge. (iii) The optical coherence induced by the laser, both in the CC and FWM situations, has an intrinsic exponential decay roughly proportional to the temperature $T$. At zero $T$, the intrinsic decay follows the well-known power law associated to the FES in the linear response. ${ }^{1}$

FES can be understood in a model of spinless free electrons, which only interact with a photoexcited hole. ${ }^{1,15,17}$ Within the Nozières-De Dominicis scheme we consider a localized hole and a contact interaction:

$$
H=\sum_{k=0}^{k_{D}} \epsilon_{k} a_{k}^{\dagger} a_{k}+\left(E_{g}+\epsilon_{F}\right) d^{\dagger} d+\frac{V}{N} \sum_{k, k^{\prime}}^{k_{D}} a_{k}^{\dagger} a_{k^{\prime}} d^{\dagger} d,
$$

where $d^{\dagger}$ creates a localized hole and $\epsilon_{k}$ is the dispersion relation of electrons at the conduction band, created by $a_{k}^{\dagger}$. $k_{D}$ is a wave-vector cutoff, $V$ the attractive potential between the hole and the electrons in the conduction band, and $N$ the linear size of the system. It must be stressed that two different kind of excitations appear in the Hamiltonian (1): the valence hole, and the conduction electron-hole pairs, which can be described, close to the Fermi energy, as bosonic excitations. ${ }^{4}$ These conduction electron-hole pairs are totally 
unrelated to the excitons in undoped semiconductors, which involve both the conduction and the valence band.

We discuss now some of the approximations involved in Hamiltonian (1). First of all, we assume that the valence hole has an infinite mass and it does not recoil in its interaction with the conduction electrons. Considering a finite mass hole would render extremely difficult an analytical calculation of the transient nonlinear response. In general, holes have a finite mass in real semiconductors. However, there is a number of situations in which the hole can behave as an infinite mass particle. Strong localization of the holes can happen due to both alloy fluctuations in general and single monolayer fluctuations in narrow quantum wells. The hole is also strongly localized in the case of "acceptor to conductionband" transitions in an $n$-doped semiconductor slightly compensated with acceptor impurities like Beryllium. ${ }^{3}$ From the theory point of view, it is well established that the finite mass of the valence hole reduces the FES, especially in emission. ${ }^{16}$ Hence, the experimental observation of FES in a real system supports the existence of strongly localized valence holes.

Second, Hamiltonian (1) only includes a single valence hole. This is known to give the correct linear response in semiconductor samples. Nevertheless, in the case of excitons, two valence hole states must be included in order to get the correct third-order optical response, ${ }^{18}$ which has a contribution coming from the exciton-exciton interaction. Note, however, that Eq. (1) is analogous to a two-level system (the valence hole) dressed by the final-state interaction with the Fermi sea electrons. This implies that the single valence hole case presents optical nonlinearities that do not exist in the excitonic case, and govern the nonlinear response in a low excitation regime. The most important process that invalidates this approximation is the overlap between the different perturbations induced on the conduction electrons by valence holes at different sites. The range of this perturbation can be estimated as $k_{F}^{-1}$. The overlap will be negligible if the density of photoexcited valence holes $\left(n_{v h}\right)$ is low enough, so that the distance between valence holes is greater than $k_{F}^{-1}$. This is the case for typical excitation densities of $10^{9}$, $10^{10} \mathrm{~cm}^{-2}$ in FWM experiments in doped GaAs quantum wells with a Fermi energy of $20 \mathrm{meV}$, so that $k_{F}^{-1} n_{v h}^{1 / 2}$ $\approx 10^{-2}$. In this range the Coulomb interaction between carriers at different valence hole sites can also be neglected. Under these conditions, the optical response of a sample with many valence holes will be equivalent to the optical response of Hamiltonian (1).

This paper is organized as follows: In Sec. II we review the bosonization approach to the FES linear response. Our original contribution starts in Sec. IIC, where we use this approach to obtain the nonlinear optical response of the FES. In Sec. III we discuss the predictions of the linear-response theory at finite temperature in the case of a $\mathrm{CC}$ experiment. In Sec. IV we apply our calculation of $\chi^{(3)}$ to the case of various FWM experiments. The discussion of our results is made in Sec. V, where we consider the comparison of the dephasing mechanisms contained in Eq. (1) with other competing processes.

\section{THEORETICAL FRAMEWORK}

\section{A. The bosonization scheme}

Since only states close to the Fermi level are excited, we can approximate the dispersion relation by $\epsilon_{k}=\left(k-k_{F}\right) / \rho$ with $\rho$ being the density of single-particle states at the Fermi level. We consider a hole potential $V$ isotropic and weak, so that only $s$-wave scattering is important. Under these conditions, the problem becomes that of one-dimensional electrons with linear energy dispersion. The bosonization approach allows us to express all the physics in terms of the bosonic fields

$$
b_{k}^{\dagger}=\sum_{k^{\prime}=k}^{k_{D}} a_{k^{\prime}}^{\dagger} a_{k^{\prime}-k} / \sqrt{k N}
$$

with $0 \leqslant k \leqslant k_{F}$. The set of operators $b_{k}, b_{k}^{\dagger}$ satisfies bosonic commutation relations only when one is restricted to the lowenergy range. ${ }^{1}$ We define $H_{i}$ as the initial Hamiltonian without a valence hole $\left(d^{\dagger} d=0\right)$ and $H_{f}$ as the final Hamiltonian after the photoexcitation of the valence hole $\left(d^{\dagger} d=1\right)$. They can be written in terms of the bosonic operators: ${ }^{4}$

$$
\begin{gathered}
H_{i}=\sum_{k} \frac{k}{\rho} b_{k}^{\dagger} b_{k}, \\
H_{f}=\omega_{0}+\sum_{k} \frac{k}{\rho}\left(b_{k}^{\dagger}+\frac{\rho V}{\sqrt{k N}}\right)\left(b_{k}+\frac{\rho V}{\sqrt{k N}}\right),
\end{gathered}
$$

where $\omega_{0}=E_{g}+\epsilon_{F}-(V \rho)^{2} \epsilon_{F}$ is the renormalized hole energy (we set $\hbar=1$ ). The index $k$ in $b_{k}^{\dagger}, b_{k}$ always runs between 0 and $k_{F}$.

$H_{i}$ and $H_{f}$ are related by a canonical transformation that describes the effect of the potential created by the valence hole onto the conduction electrons:

$$
H_{f}=\omega_{0}+U^{\dagger} H_{i} U,
$$

where

$$
U=\exp \left[V \rho \sum_{k} \frac{1}{\sqrt{k N}}\left(b_{k}^{\dagger}-b_{k}\right)\right] .
$$

Optical properties are determined from the adequate correlation functions of the electric dipole operator $P^{\dagger}$ $=\mu \quad a^{\dagger} d^{\dagger}$, where $\mu$ is the dipole matrix element and $a^{\dagger}$ is the creation operator of conduction electrons at the localized hole site. This operator can also be expressed as an exponential of Tomonaga boson operators:

$$
a^{\dagger}=\sum_{k=0}^{k_{D}} a_{k}^{\dagger}=\exp \left[\sum_{k} \frac{1}{\sqrt{k N}}\left(b_{k}^{\dagger}-b_{k}\right)\right] .
$$

\section{B. Linear response}

The linear response $\chi^{(1)}(t)$ is given (in the rotating wave approximation) by the expression

$$
\chi^{(1)}(t)=i \theta(t)\left\langle P(t) P^{\dagger}(0)\right\rangle .
$$


$P^{\dagger}$ creates a valence hole, so that the system evolves under the final Hamiltonian $H_{f}$ in the interval $(0, t)$ :

$$
\begin{aligned}
\left\langle P(t) P^{\dagger}(0)\right\rangle & =\left\langle e^{i H t} P(0) e^{-i H t} P^{\dagger}(0)\right\rangle \\
& =\left\langle e^{i H_{i} t} a U^{\dagger} e^{-i H_{i} t} U a^{\dagger}\right\rangle e^{-i \omega_{0} t} \\
& =\left\langle B(t) B^{\dagger}(0)\right\rangle e^{-i \omega_{0} t},
\end{aligned}
$$

where

$$
B^{\dagger}(t)=\exp \left[(1+V \rho) \sum_{k} \frac{1}{\sqrt{k N}}\left(b_{k}^{\dagger} e^{i \frac{k}{\rho} t}-b_{k} e^{-i \frac{k}{\rho} t}\right)\right] .
$$

The original Schotte and Schotte result ${ }^{4}$ can be extended to the case of nonzero temperature by considering a bath of Tomonaga bosons at thermal equilibrium in the average (8):

$$
\begin{aligned}
\left\langle B(t) B^{\dagger}(0)\right\rangle= & \exp \left\{-\sum_{k} \frac{(1+V \rho)^{2}}{k N}\left\{\left[1+2 N_{B}(k)\right]\right.\right. \\
& \left.\left.\times\left[1-\cos \left(\frac{k}{\rho} t\right)\right]+i \sin \left(\frac{k}{\rho} t\right)\right\}\right\},
\end{aligned}
$$

where $N_{B}(k)$ is the Bose-Einstein occupation factor. We are interested in the long-time limit of the response functions. The cutoff in momentum space in Eq. (10) is $k_{F}$ and it implies a cutoff in energy space, $\epsilon_{c}=k_{F} / \rho=2 \epsilon_{F}$, as usually taken in the bosonization procedure. In the limit $t \gg \epsilon_{c}^{-1}$ we obtain

$$
\chi^{(1)}(t)=i \mu^{2} \theta(t)\left[i \epsilon_{c} \frac{\sinh \left(\pi k_{B} T t\right)}{\pi k_{B} T}\right]^{-\alpha} e^{-i \omega_{0} t},
$$

where $\alpha=(1+V \rho)^{2}$. Expression (11) will be valid in the case of near-resonance excitation and spectrally narrow pulses, that is, $\left|\omega-\omega_{0}\right| \ll \epsilon_{c}$, and $(\Delta t)^{-1} \ll \epsilon_{c}(\omega$ is the excitation energy). Condition $k_{B} T \ll \epsilon_{c}$ must also be fulfilled in order to consider low-energy excitations only. At zero $T$, Eq. (11) recovers the well-known behavior $\chi^{(1)}(t)$ $=i \mu^{2} \theta(t)\left(i \epsilon_{c} t\right)^{-\alpha}$.

In the spectral domain, the absorption is given by $\theta(\omega$ $\left.-\omega_{0}\right)\left(\omega-\omega_{0}\right)^{(\alpha-1)}$ so that FES takes place for $\alpha<1$. In the time domain, the FES is characterized by the intrinsic power-law decay of the response function (with $\alpha<1$ ). As we show below, the decay of the optical coherence, i.e., the dephasing, is an increasing function of $\alpha$, which is the square of a sum of two terms that have different physical origin and opposite effects. The first term 1, is related to the addition of a new electron to the Fermi level in the absorption process. The second term $-|V \rho|$, is related to the sudden switching of the hole potential. The first term makes dephasing more efficient while the second one makes dephasing less efficient.

\section{Third-order susceptibility}

To study the nonlinear response of the electron gas we concentrate on FWM experiments, which are usually described by means of the third-order susceptibility $\chi^{(3)}$. However it is not evident whether a perturbative expansion in terms of the electric field is justified in the case of the nonlinear optical response of Hamiltonian (1). Primozich et al. ${ }^{10,11}$ have shown the validity of such an expansion provided that $\left(\mu \mathcal{E}_{0} \Delta t\right)^{2} \ll 1$. Considering excitation intensities of $\mathrm{mW}, \Delta t=0.7 \mathrm{ps}$, and known values for the interband dipole matrix element of GaAs, ${ }^{19}$ one obtains $\left(\mu \mathcal{E}_{0} \Delta t\right)^{2}$ $\approx 10^{-3}$. Thus, we can consider terms up to the third order in the electric field for the ultrafast transient experiments described below.

We consider the typical situation in which the system is excited by two mutually delayed laser pulses that propagate along different directions, $\mathbf{k}_{\mathbf{1}}$ and $\mathbf{k}_{\mathbf{2}}$, with $\left|\mathbf{k}_{\mathbf{1}}\right|=\left|\mathbf{k}_{\mathbf{2}}\right|$. In any system with translational invariance and some degree of nonlinearity in the optical response, these exciting pulses will induce an electric dipole that will re-emit light along the direction $2 \mathbf{k}_{\mathbf{2}}-\mathbf{k}_{\mathbf{1}}$. Up to the third order in the external field, the FWM signal is given by

$$
\begin{aligned}
F_{F W M}(t)= & \int_{-\infty}^{t} d t_{1} d t_{2} d t_{3} \chi^{(3)}\left(t-t_{1}, t-t_{2}, t-t_{3}\right) \\
& \times E_{1}^{*}\left(t_{2}\right) E_{2}\left(t_{1}\right) E_{2}\left(t_{3}\right)+\text { H.c. }
\end{aligned}
$$

where $E_{1,2}$ are the electric fields in the directions $\mathbf{k}_{\mathbf{1 , 2}}$. As in the case of CC, FWM takes place as long as the polarization induced by the first laser pulse is not wiped out before the second pulse reaches the sample. For this reason, both CC and FWM can be used to measure $T_{2}$. In undoped samples $\chi^{(3)}$ is related to the exciton-exciton interaction. In the case of the FES we are going to see that $\chi^{(3)}$ is not zero even for noninteracting electrons. This constitutes an important difference between the doped and undoped systems.

Performing a perturbation expansion up to third order in the electric field, it can be shown that $\chi^{(3)}$ is proportional to the average of four polarization operators: ${ }^{20}$

$$
\begin{aligned}
\chi^{(3)}\left(t-t_{1}, t-t_{2}, t-t_{3}\right)= & -i\left[\theta\left(t-t_{1}\right) \theta\left(t_{1}-t_{2}\right) \theta\left(t_{2}-t_{3}\right)\right. \\
& \times\left\langle P(t) P^{\dagger}\left(t_{1}\right) P\left(t_{2}\right) P^{\dagger}\left(t_{3}\right)\right\rangle \\
& +\theta\left(t-t_{1}\right) \theta\left(t_{1}-t_{2}\right) \theta\left(t-t_{3}\right) \\
& \left.\times\left\langle P\left(t_{2}\right) P^{\dagger}\left(t_{1}\right) P(t) P^{\dagger}\left(t_{3}\right)\right\rangle\right] .
\end{aligned}
$$

In $\left\langle P(t) P^{\dagger}\left(t_{1}\right) P\left(t_{2}\right) P^{\dagger}\left(t_{3}\right)\right\rangle$ the second and fourth polarization operators create a valence hole, so that the system evolves under $H_{f}$ inside the intervals $\left(t_{2}, t_{3}\right)$ and $\left(t, t_{1}\right)$. Using the same argument that leads to Eq. (8), it is straightforward to show that

$$
\begin{aligned}
\left\langle P(t) P^{\dagger}\left(t_{1}\right) P\left(t_{2}\right) P^{\dagger}\left(t_{3}\right)\right\rangle= & \mu^{4}\left\langle B(t) B^{\dagger}\left(t_{1}\right) B\left(t_{2}\right) B^{\dagger}\left(t_{3}\right)\right\rangle \\
& \times \exp \left[-i\left(t-t_{1}+t_{2}-t_{3}\right) \omega_{0}\right] .
\end{aligned}
$$

Using the definition of $B^{\dagger}$ given by Eq. (9), we can express $\chi^{(3)}$ as the thermal average of a product of four exponentials of bosons. The average of a product of any number of exponentials of bosons can be factorized into twoexponential correlation functions. In Appendix A this fact is used to prove the general result: 


$$
\begin{gathered}
\left\langle B\left(t_{0}\right) B^{\dagger}\left(t_{1}\right) \ldots B\left(t_{n-1}\right) B^{\dagger}\left(t_{n}\right)\right\rangle \\
=\prod_{j>i=0}^{n}\left\langle B\left(t_{i}\right) B^{\dagger}\left(t_{j}\right)\right\rangle^{(-1)^{1+i+j}},
\end{gathered}
$$

where $n$ is an odd integer. $\left\langle B\left(t_{i}\right) B^{\dagger}\left(t_{j}\right)\right\rangle$ is given by Eq. (10) and, in the long-time approximation, by Eq. (11). The nonlinear susceptibility $\chi^{(n)}$ at any order $n$ can be expressed by means of products of $n+1$ polarization operators of the form $\left\langle P P^{\dagger} \ldots P P^{\dagger}\right\rangle$. Each polarization operator can be expressed as an exponential of bosonic operators. Thus, Eq. (15) allows the calculation of the optical response of the FES at any order in the electric field in the long-time limit, under the approximations discussed in Sec. I.

Application of Eq. (15) to the case of $\chi^{(3)}$ yields the result

$$
\left\langle P(t) P^{\dagger}\left(t_{1}\right) P\left(t_{2}\right) P^{\dagger}\left(t_{3}\right)\right\rangle=\mu^{4} \frac{\left\langle B(t) B^{\dagger}\left(t_{1}\right)\right\rangle\left\langle B\left(t_{1}\right) B^{\dagger}\left(t_{2}\right)\right\rangle\left\langle B\left(t_{2}\right) B^{\dagger}\left(t_{3}\right)\right\rangle\left\langle B(t) B^{\dagger}\left(t_{3}\right)\right\rangle}{\left\langle B(t) B^{\dagger}\left(t_{2}\right)\right\rangle\left\langle B\left(t_{1}\right) B^{\dagger}\left(t_{3}\right)\right\rangle} \exp \left[-i\left(t-t_{1}+t_{2}-t_{3}\right) \omega_{0}\right] .
$$

This result implies that $\chi^{(3)}$ will present singularities similar to that of $\chi^{(1)}$. Using the result of Eq. (11) in Eq. (16) we obtain the following $T=0$ expression for $\chi^{(3)}$ :

$$
\chi^{(3)} \propto\left[\frac{\left(t-t_{1}\right)\left(t_{1}-t_{2}\right)\left(t_{2}-t_{3}\right)\left(t-t_{3}\right)}{\left(t-t_{2}\right)\left(t_{1}-t_{3}\right)}\right]^{-\alpha} .
$$

This simple expression is valid for $0 \leqslant \alpha<1$. Out of this range the expression is more complicated. Equations (12), (13), and (16) allow us to calculate the FWM signal in nearresonance experiments, under the same conditions explained under Eq. (11).

\section{LINEAR RESPONSE: COHERENT CONTROL EXPERIMENTS}

In CC experiments, the sample is excited by a pair of phase-locked identical laser pulses delayed in a time $\tau$ with respect to each other. The total energy absorbed by the system $W$, as a function of the delay $\tau$, can be measured by detecting the reflectivity changes produced by the photoexcitation density ${ }^{12}$ or by measuring the total luminiscense emitted by the sample. ${ }^{13}$ These experiments are carried out in the linear- regime, where the total energy absorbed after photoexcitation can be easily calculated by means of the linearresponse function:

$$
W(\tau)=2 \operatorname{Im} \int_{-\infty}^{\infty} \chi^{(1)}\left(t_{1}-t_{2}\right) E^{*}\left(t_{1}\right) E\left(t_{2}\right) d t_{1} d t_{2} .
$$

The electric field of the phase-locked laser pulses is given by $E(t)=\mathcal{E}(t) e^{-i \omega_{0} t}+\mathcal{E}(t-\tau) e^{-i \omega_{0}(t-\tau)}$. The pulses are thus spectrally peaked around the FES transition. The envelope functions are Gaussian pulses of width $\Delta t: \mathcal{E}(t)$ $=\mathcal{E}_{0} e^{-t^{2} / \Delta t^{2}}$. Substituting the electric field into the expression (18) it can be clearly seen that $W(\tau)$ depends strongly on $\tau$. It oscillates with frequency $\omega_{0}$, showing that the absorption in doped semiconductors, close to a Fermi-edge singularity, can be coherently controlled. The phase and the amplitude of these oscillations change also with $\tau$. We can distinguish three different regimes: (i) For $\tau<\Delta t$, the two pulses overlap: the absorbed energy oscillates between 0 (destructive interference) and $4 W_{S P}$ (constructive interference)

$$
W(\tau)=2 W_{S P}\left[1+\cos \left(\omega_{0} \tau\right)\right]
$$

where $W_{S P}$ is the energy transfered by a single pulse.

(ii) For $\tau \gg \Delta t, 1 / \pi k_{B} T$, the decay of the polarization between the two pulses is exponential, as can be seen clearly in the behavior of $\chi^{(1)}$ for long times. It can be easily proved that in this regime

$$
W(\tau)=2 W_{S P}+W_{C C} e^{-\alpha \pi k_{B} T \tau} \cos \left(\omega_{0} \tau+\alpha \frac{\pi}{2}\right),
$$

where $W_{C C}$ is the constant prefactor before the exponential decay and is given by

$$
W_{C C}=2 \pi(\Delta t)^{2}\left(\frac{\epsilon_{c}}{2 T}\right)^{-\alpha} \exp \left[\frac{1}{2}\left(\alpha \Delta t k_{B} T\right)^{2}\right] .
$$

In the general case $W_{C C} \neq 2 W_{S P}$, due to the finite width of the exciting pulses and the fact that the decay is nonexponential for short times. Equation (20) shows important differences with the case of $\mathrm{CC}$ of excitons. First of all, a phase shift of $\alpha \pi / 2$ appears in the CC oscillations at long $\tau$. This surprising behavior is not observed in undoped samples, ${ }^{12}$ where the maxima of the oscillations are exactly at $\tau$ $=2 n \pi / \omega_{0}$. The great interest of this phase shift in the CC oscillations resides in the fact that it is independent of the relative importance of other competing dephasing processes. This could allow a more accurate determination of the FES exponent $\alpha$, than in continuous-wave photoluminescence experiments.

Second, the exponent of the coherence decay behaves linearly with temperature, with the factor $\pi \alpha$. In Sec. V it is shown that this one is the most important temperature dependent dephasing mechanism at low temperatures. Thus, the measure of the decay time of the CC oscillations could allow another independent determination of the singularity exponent.

(iii) For very low temperatures, we can have $1 / \pi k_{B} T \gg \tau$ $\gg \Delta t$. In this range, the decay of the polarization is nonexponential, even when the pulses do not overlap, because of 


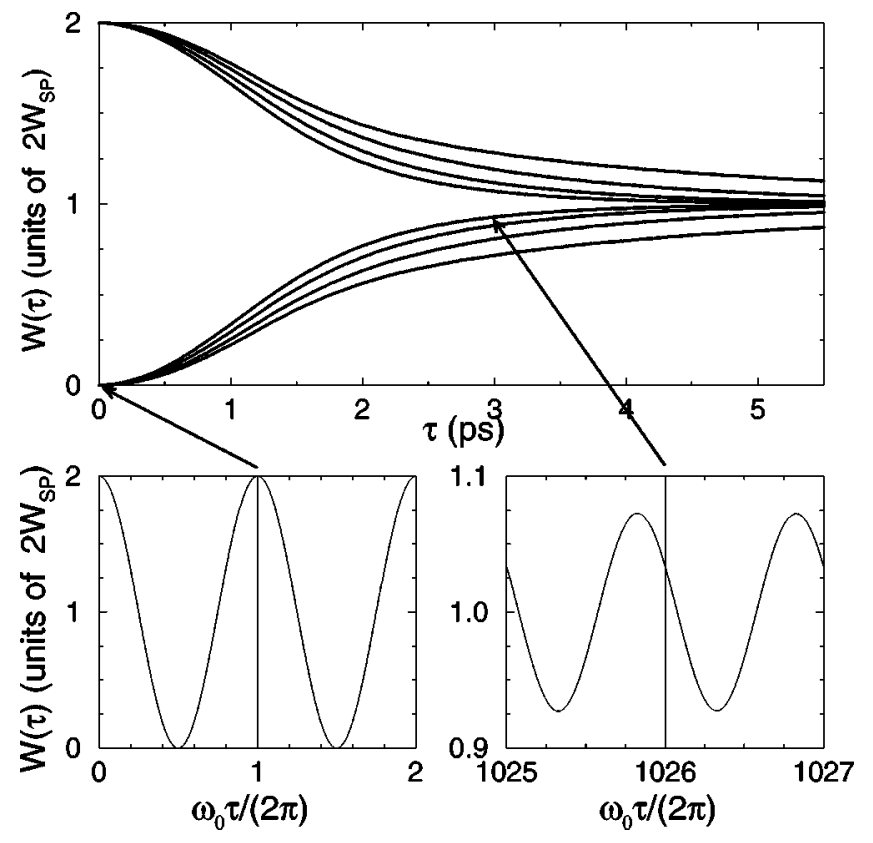

FIG. 1. Upper panel: evolution of the absorbed energy as a function of $\tau$ for Gaussian pulses of width $\Delta t=0.7 \mathrm{ps}, \alpha=0.7$, and $\omega_{0}=1.5 \mathrm{eV}$. Only the envelopes of the CC oscillations are plotted, corresponding to temperatures between $1 \mathrm{~K}$ (outer) and $4 \mathrm{~K}$ (inner). Lower panels: CC oscillations at $\tau=0$ ps (left) and $\tau=3$ ps (right) for the case $T=4 \mathrm{~K}$. In the right panel the maxima of the oscillations are not at integer values of $\omega_{0} \tau /(2 \pi)$, showing a characteristic phase shift.

the behavior of $\chi^{(1)}$ at short times. However, the condition $\tau, \Delta t>\epsilon_{c}^{-1}$ can still be fulfilled, so the asymptotic approximation that leads to Eq. (11) is valid. This nonexponential decay is another important difference with the undoped case.

In an intermediate region of parameters, the integration in Eq. (18) must be performed numerically. The result of this calculation is presented in Fig. 1 , for $T=1-4 K$, and clearly shows the different regimes and the phase shift $\alpha \pi / 2$ and exponential relaxation for long $\tau$.

The main conclusion from this section is that the absorption in doped semiconductors, close to the FES, can be coherently controlled. The decay of the polarization predicted in cases (ii) and (iii) is not produced by any inelasticscattering mechanism or some sort of inhomogeneous broadening as it happens in the $\mathrm{CC}$ of excitons. ${ }^{14}$ Instead, it is an intrinsic effect due to the excitation of a continuum of bosonic modes with a distribution of energies that implies destructive interference in the time domain. In the absence of the potential created by the photoexcited hole $(V=0)$, this effect has been described as inhomogeneous broadening in momentum space. ${ }^{6}$ However, the sudden switching of the hole potential partially compensates the effect of the momentum space broadening, reducing the dephasing. This situation resembles that of the experiment of Wehner et al., ${ }^{21}$ where the electron-LO phonon scattering rate is coherently controlled. In our case, the Tomonaga bosons play the role of the phonons in that experiment, with an important difference: the Tomanaga bosons form a gapless continuum of modes, which leads to the dephasing of the optical polarization.

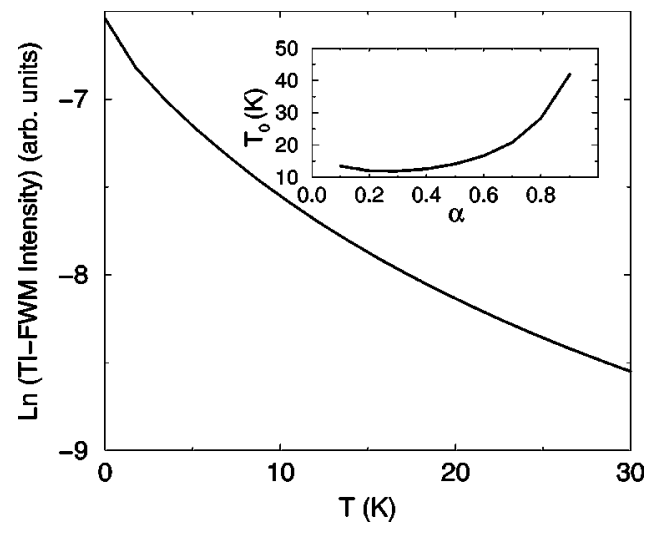

FIG. 2. Temperature dependence of the TI-FWM signal for $\alpha$ $=0.7, \tau=0$, and $\Delta t=0.7 \mathrm{ps}$. The inset shows the exponential decay parameter $T_{0}$ between 10 and $30 \mathrm{~K}$ as a function of $\alpha$.

\section{NONLINEAR RESPONSE: FOUR WAVE-MIXING EXPERIMENTS}

In this section we study the usual transient FWM experiments in which the exciting fields appearing in Eq. (12) are $E_{1,2}(t)=\mathcal{E}_{1,2}(t) e^{-i \omega t}$, where $\mathcal{E}_{1,2}(t)$ are Gaussian pulses of width $\Delta t$, delayed in $\tau$ with respect to each other $\left[\mathcal{E}_{2}(t\right.$ $\left.-\tau)=\mathcal{E}_{1}(t)\right]$, and $\omega$ is the central exciting frequency, which is taken at the FES resonance.

\section{A. Decay of the four-wave mixing intensity with temperature}

We are now interested in the properties of the nonlinear optical response, rather than in the dephasing processes between the pulses. Therefore, in Secs. IV A and IV B, we take $\tau=0$. From the factorization formula for $\chi^{(3)}$ given in Eq. (16), we expect to find, in a FWM experiment, some of the characteristics of the FES in linear response, such as a strong dependence on temperature.

In order to test this idea we calculate the time-integrated FWM (TI-FWM) intensity, $I_{F W M}=\int d t\left|F_{F W M}(t)\right|^{2}$ when the sample is excited at resonance $\left(\omega=\omega_{0}\right)$ by Gaussian pulses with $\Delta t=0.7$ ps. In Fig. 2 we present our results for the particular case $\alpha=0.7$, as a function of temperature. We focus on the interval between 10 and $30 \mathrm{~K}$, for comparison with experiments ${ }^{7}$ (at higher $T$ the condition $k_{B} T \ll \epsilon_{c}$ is not satisfied). In this range our result for the decay with temperature can be fitted to an exponential form $e^{-T / T_{0}(\alpha)}$, so that we can obtain a characteristic temperature $T_{0}(\alpha)$ that governs the decay of the FWM signal. The parameter $T_{0}$ is plotted as a function of $\alpha$ in the inset of Fig. 2. A similar exponential decay of the TI-FWM of a doped sample under high magnetic field has been observed by Bar-Ad et al. ${ }^{7}$ If we apply our zero magnetic field theory to their result, we would infer $\alpha \approx 0.7$, a good value to get FES as the ones observed in continuous-wave spectroscopy. ${ }^{2}$ This could be a hint that the physics of the FES under magnetic fields could be described by a model similar to the one presented here, but further work is needed to clarify this point.

\section{B. Four-wave mixing intensity as a function of the exciting frequency}

Now we treat the case in which $\tau=0$ and the exciting pulses are slightly out of resonance $\left(\omega \neq \omega_{0}\right)$. Condition $\mid \omega$ 


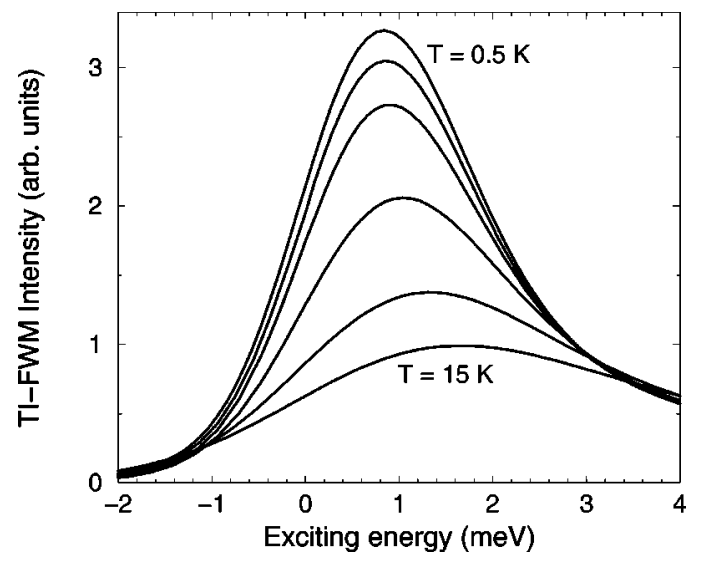

FIG. 3. TI-FWM signal as a function of the exciting frequency $\omega-\omega_{0}$, for the case $\Delta t=0.7 \mathrm{ps}, \alpha=0.7$, and different temperatures $T=0.5,1,2,5,10$, and $15 \mathrm{~K}$ (from top to bottom).

$-\omega_{0} \mid \ll \epsilon_{c}$ must be satisfied in order for the bosonization procedure to be valid. We have calculated the TI-FWM intensity as a function of $\omega$ for $\Delta t=0.7 \mathrm{ps}, \alpha=0.7$, and different temperatures. Our results are presented in Fig. 3, where it is shown that the FES appears as an asymmetric resonance in the FWM spectrum, similar to the one which is observed in linear spectroscopy. The FES resonance is strongly suppressed with temperature and shows Lorentzian broadening for high $T$, as expected from the exponential decay of $\chi^{(3)}$ at long times. This fact allows us to unambiguously determine the observation of the FES in the nonlinear regime. A strong resonance in the FWM signal as a function of the exciting frequency was reported in the work of Kim et al., ${ }^{6}$ in a doped sample, which also showed a FES resonance in the photoluminescence experiments.

\section{Four-wave mixing signal as a function of $\tau$}

In a transient degenerate FWM experiment the nonlinear signal can be studied as a function of the time delay, $\tau$. We consider first the time resolved FWM (TR-FWM) signal, $F_{F W M}(\tau, t)$, which is a function of both the detection time $t$ and the delay $\tau$. $F_{F W M}(\tau, t)$ can be estimated by assuming that the laser-pulse amplitudes can be approximated by $\delta$ functions (obviously, this is justified in the case $t \gg \tau \gg \Delta t$ ). Using this assumption, simple analytical expressions can be obtained:

$$
\begin{aligned}
F_{F W M}(\tau, t) \propto & -i \mu^{4}\left(i \epsilon_{c}\right)^{-3 \alpha} \theta(\tau) \theta(t-\tau) e^{-i \omega_{0} t} \\
& \times\left[\frac{\sinh ^{2}\left(\pi k_{B} T \tau\right) \sinh ^{2}\left[\pi k_{B} T(t-\tau)\right]}{\left(\pi k_{B} T\right)^{3} \sinh \left(\pi k_{B} T t\right)}\right]^{-\alpha}+\text { H.c. }
\end{aligned}
$$

For large $t, F_{F W M}(t)$ presents an exponential decay exp $\left[-\alpha \pi k_{B} T t\right]$ which becomes a power-law decay $t^{-\alpha}$ at zero temperature. $^{22}$

Usually, the TI-FWM intensity as a function of $\tau$, $I_{F W M}(\tau)=\int d t\left|F_{F W M}(\tau, t)\right|^{2}$, is measured in the experiments. ${ }^{7}$ In order to obtain realistic results beyond the deltalike pulses approximation, we have performed numerical integrations of Eq. (12) with Gaussian pulses having $\Delta t$

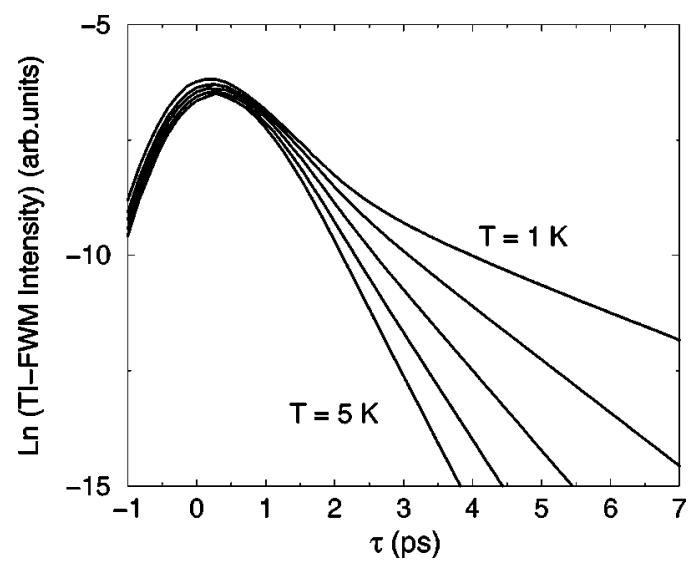

FIG. 4. TI-FWM signal as a function of $\tau$ (in ps) for $\alpha=0.7$ and $\Delta t=0.7 \mathrm{ps}$ at different temperatures from 1 up to $5 \mathrm{~K}$ by successively increasing $T$ in $1 \mathrm{~K}$ steps.

$=0.7$ ps and $\alpha=0.7$ as shown in Fig. 4, for different temperatures. The maximum is located around $\tau=0$, for which the overlap of the laser pulses is maximum. $I_{F W M}(\tau)$ can show nonexponential relaxation for $1 / \pi k_{B} T>\tau \gg \Delta t$, in exact analogy to the case of the dephasing of CC oscillations discussed in Sec. III. For $\tau \gg \Delta t, 1 / \pi k_{B} T$, it can be analytically shown from our calculation of $\chi^{(3)}$ that the decay is exponential, of the form $e^{-2 \alpha \pi k_{B} T \tau}$. It must be pointed out that the two different regimes of the TI-FWM as a function of $\tau$ shown in Fig. 4 have been observed experimentally ${ }^{23}$ in the presence of a magnetic field.

\section{DISCUSSION AND CONCLUSION}

The main concern of this paper is the temporal evolution of the laser induced optical coherence of a doped semiconductor in the regime where FES is observed. In marked contrast with undoped semiconductors, the induced coherence decays, even at zero temperature, without the intervention of any inelastic scattering or statistical broadening. We refer to this decay as intrinsic dephasing. Its origin lies in the excitation of a continuum of low-energy conduction electronhole pairs whenever a hole is promoted from the valence band to the conduction band. In the spectral domain, these low-energy excitations can give rise to the FES. In the time domain they produce the intrinsic dephasing.

We have presented calculations of the optical response of a doped semiconductor, as modeled by Hamiltonian (1), in some standard experimental situations. The question is whether the physical processes not included in that Hamiltonian will obscure our predictions. There are three additional sources of decay of the optical coherence that can compete with the "intrinsic dephasing:' electron-electron $(e-e)$ scattering, electron-phonon scattering, and inhomogeneous broadening of the localized valence hole levels. ${ }^{5}$

A rough estimate of the decay time of the optical coherence due to $e-e$ scattering, $T_{2}^{e-e}$, can be obtained as the inverse of the scattering rate of electrons at $\epsilon_{F}$. For twodimensional electrons it has been $\operatorname{shown}^{24}$ that $\left(T_{2}^{e-e}\right)^{-1}$ $\propto T^{2} \log (T)$, at $k_{B} T \ll \epsilon_{F}$. At low $T$, this $e$-e dephasing is less important than the FES intrinsic dephasing (linear in $T$ ). Em- 
ploying a Thomas-Fermi approximation, we can estimate $T_{2}^{e-e}=15 \mathrm{ps}$ at $10 \mathrm{~K}$ and $\epsilon_{F}=20 \mathrm{meV}$, showing a dephasing much slower than $T_{2}^{F E S}=1 / \alpha \pi k_{B} T=0.35 \mathrm{ps,} \mathrm{for} \alpha=0.7$ and the same temperature. The effect of electron-electron interaction in the nonlinear optical response of doped samples has been considered in more detail by Primozich et al. ${ }^{10,11}$ for pump-probe experiments, where $\left|\mathcal{E}_{2}\right| \gg\left|\mathcal{E}_{1}\right|$.

The electron-phonon interaction will also have a contribution, mainly due to the scattering between conduction electrons and acoustical phonons, which are the relevant lattice excitations at low energies. This interaction can be described by a deformation potential Hamiltonian, ${ }^{1}$ which implies a cubic dependence on temperature of the scattering rate $\left(T_{2}^{p h}\right)^{-1} \propto T^{3}$. We have performed an estimate of this dephasing time, which yields $\left(T_{2}^{p h}\right)^{-1}=80 \mathrm{~ns}$ for an electron at $\epsilon_{F}=20 \mathrm{meV}, T=10 \mathrm{~K}$, in a GaAs quantum well.

Thus, both electron-electron and electron-phonon effects give rise to slower decays of the optical coherence so that they will not compete with the FES intrinsic dephasing at low temperatures.

The decay of the optical coherence due to the broadening in the distribution of the hole energies depends on the particular details of each sample. However, this dephasing is quite independent of temperature. In the case in which inhomogeneous broadening is more efficient than intrinsic FES dephasing, the experimental study of $\left(T_{2}\right)^{-1}$ as a function of temperature would allow to separate the linear term, $\left(T_{2}^{F E S}\right)^{-1}$, which is the most important temperaturedependent contribution, as we have shown.

Hence, it is our contention that the dynamics of the optical coherence of a doped sample in the FES regime, as described in this paper, can be observed. However, the limitations of both the Hamiltonian, the bosonization, and the perturbative expansion call for further work on the theory side.

From the experimental point of view, the realization of the experiments suggested in this paper would permit independent measurement of the singularity exponent $\alpha$, as well as the observation of new physical phenomena, like the phase shift in the CC oscillations (Sec. III) or the FES in the time integrated FWM signal as a function of the exciting frequency near the Fermi energy.

In summary, we have presented a theory for the transient optical response of the FES. The use of the bosonization to describe the low-energy excitations across the Fermi level allows the analytical evaluation of the linear and nonlinear response both at zero and finite temperature. $\mathrm{CC}$ of the energy absorbed at resonance with the FES can be performed. CC oscillations show a phase shift that depends on the singularity exponent $\alpha$. The FWM signal shows a sharp asymmetric resonance near $\epsilon_{F}$ as a function of the exciting energy, and is strongly suppressed with temperature. We have shown that both CC and FWM experiments could be used to study the decay of the laser induced coherence or dephasing. In contrast to the case of undoped samples, the bath of Tomonaga bosons responsible for the FES produces a new dephasing mechanism that depends linearly on temperature.

\section{ACKNOWLEDGMENTS}

This work supported in part by MEC of Spain under Contract No. PB96-0085, Fundacion Ramón Areces and CAM under Contract No. 07N/0026/1998. D.P. thanks the Spanish Education Ministry for its FPU grant. J. F.-R. acknowledges the Spanish Ministry of Education for its financial support.

\section{APPENDIX A: GENERAL EXPRESSION FOR THE AVERAGE OF $N$ POLARIZATION OPERATORS}

In this appendix we prove Eq. (15), which allows us to calculate the nonlinear optical susceptibilities at any order $n$. First of all we factorize the correlation function into different bosonic modes:

$$
\begin{aligned}
& \left\langle B\left(t_{0}\right) B^{\dagger}\left(t_{1}\right) \ldots B\left(t_{n-1}\right) B^{\dagger}\left(t_{n}\right)\right\rangle \\
& \quad=\prod_{k}\left\langle B_{k}\left(t_{0}\right) B_{k}^{\dagger}\left(t_{1}\right) \ldots B_{k}\left(t_{n-1}\right) B_{k}^{\dagger}\left(t_{n}\right)\right\rangle,
\end{aligned}
$$

where $\quad B_{k}^{\dagger}(t)=\exp \left[\beta_{k}^{*}(t) b_{k}^{\dagger}-\beta_{k}(t) b_{k}\right], \quad$ with $\quad \beta_{k}(t)=(1$ $+V \rho) e^{i(k / \rho) t}$. We ignore for the moment the index $k$ and define $\beta_{j}=\beta\left(t_{j}\right)$. Using the well-known relation $e^{A} e^{B}$ $=e^{A+B} e^{\frac{1}{2}[A, B]}$ we can easily show that

$$
\begin{aligned}
& \left\langle B\left(t_{0}\right) B^{\dagger}\left(t_{1}\right) \ldots B\left(t_{n-1}\right) B^{\dagger}\left(t_{n}\right)\right\rangle \\
& =\prod_{j>i=0}^{n} \exp \left[-i \operatorname{Im}\left(\beta_{i}^{*} \beta_{j}\right)(-1)^{i+j+1}\right] \\
& \quad \times\left\langle\exp \left\{-\sum_{i}\left[(-1)^{i} \beta_{i}^{*} b^{\dagger}-\text { H.c. }\right]\right\}\right) .
\end{aligned}
$$

The average in Eq. (A2) is calculated assuming a thermal distribution of bosons:

$$
\begin{aligned}
& \left\langle\exp \left\{-\sum_{i}\left[(-1)^{i} \beta_{i}^{*} b^{\dagger}-\text { H.c. }\right]\right\}\right\rangle \\
& \quad=\exp \left\{-\left[1 / 2+N_{B}(k)\right]\left|\sum_{j} \beta_{j}(-1)^{j}\right|^{2}\right\} .
\end{aligned}
$$

We expand the absolute value inside of the exponential in Eq. (A3):

$$
\left|\sum_{j} \beta_{j}(-1)^{j}\right|^{2}=2 \sum_{j>i}\left[|\beta|^{2}-\operatorname{Re}\left(\beta_{i}^{*} \beta_{j}\right)\right](-1)^{i+j+1} .
$$

We have $\operatorname{Re}\left(\beta_{i}^{*} \beta_{j}\right)=(1+V \rho)^{2} \cos (k / \rho)\left(t_{i}-t_{j}\right) \quad$ and $\operatorname{Im}\left(\beta_{i}^{*} \beta_{j}\right)=(1+V \rho)^{2} \sin (k / \rho)\left(t_{i}-t_{j}\right)$. Substituting Eq. (A3) into Eq. (A2), and writing explicitly the momentum index $k$, we obtain 


$$
\begin{aligned}
& \left\langle B\left(t_{0}\right) B^{\dagger}\left(t_{1}\right) \ldots B\left(t_{n-1}\right) B^{\dagger}\left(t_{n}\right)\right\rangle \\
& =\prod_{j>i=0}^{n}\left[\operatorname { e x p } \left(-\alpha \sum_{k}\left\{\left[1+2 N_{B}(k)\right]\right.\right.\right. \\
& \left.\left.\left.\left.\quad \times\left[1-\cos \frac{k}{\rho}\left(t_{i}-t_{j}\right)\right]+i \sin \frac{k}{\rho}\left(t_{i}-t_{j}\right)\right\}\right)\right]\right]^{(-1)^{i+j+1}} \\
& =\prod_{j>i=0}^{n}\left\langle B\left(t_{i}\right) B^{\dagger}\left(t_{j}\right)\right\rangle^{(-1)^{i+j+1}} .
\end{aligned}
$$

The factorization formula implies that $\chi^{(n)}$ of Hamiltonian (1) can be expressed as a product of linear $\left(\chi^{(1)}\right)$ susceptibilities, when one restricts to the low-energy spectrum (that is, resonant excitation at the FES and long-time response). A very similar factorization is found in other physical problems in which a localized level interacts with the low-energy excitations of an electron bath, such as the Kondo effect ${ }^{25}$ or an impurity in a Luttinger liquid. ${ }^{26}$ In both cases, the factorization formula allows us to write a perturbation expansion in a parameter that plays the role of the electric field in the FES case.
*Present address: Department of Physics. University of California San Diego, La Jolla, CA 92092.

${ }^{1}$ G.D. Mahan, Many-Particle Physics (Plenum, New York, 1981).

${ }^{2}$ M.S. Skolnick, J.M. Rorison, K.J. Nash, D.J. Mowbray, P.R. Tapster, S.J. Baas, and A.D. Pitt, Phys. Rev. Lett. 58, 2130 (1987); I.V. Kukushkin, K. von Klitzing, and K. Ploog, Phys. Rev. B 37, 8509 (1989); W. Chen, M. Fritze, W. Walecki, A.V. Nurmikko, D. Ackley, J.M. Hong, and L.L. Chang, ibid. 45, 8464 (1992); K.J. Nash, M.S. Skolnick, M.K. Saker, and S.J. Bass, Phys. Rev. Lett. 70, 3115 (1993); J. Rubio, H.P. van der Meulen, J.M. Calleja, R. Bergmann, V. Häautrle, and F. Scholz, Phys. Rev. B 55, 16390 (1997); S.A. Brown, J.F. Young, Z. Wasilewski, and P.T. Coleridge, ibid. 56, 3937 (1997).

${ }^{3}$ H.P. van der Meulen, I. Santa-Olalla, J. Rubio, J.M. Calleja, K.J. Friedland, R. Hey, and K. Ploog, Phys. Rev. B 60, 4897 (1999).

${ }^{4}$ K.D. Schotte and U. Schotte, Phys. Rev. 182, 479 (1969).

${ }^{5}$ J. Shah, Ultrafast Spectroscopy of Semiconductors and Semiconductor Nanostructures (Springer-Verlag, Berlin, 1996).

${ }^{6}$ D.-S. Kim, J. Shah, J.E. Cunningham, T.C. Damen, S. SchmittRink, and W. Schafer, Phys. Rev. Lett. 68, 2838 (1992).

${ }^{7}$ S. Bar-Ad, I. Bar-Joseph, Y. Levinson, and H. Shtrikman, Phys. Rev. Lett. 72, 776 (1994).

${ }^{8}$ I. Brener, W.H. Knox, and W. Schäffer, Phys. Rev. B 51, 2005 (1995).

${ }^{9}$ I.E. Perakis and D.S. Chemla, Phys. Rev. Lett. 72, 3202 (1994).

${ }^{10}$ N. Primozich, T. V. Shahbazyan, I. E. Perakis, and D.S. Chemla, Phys. Rev. B 61, 2041 (2000).

${ }^{11}$ I.E. Perakis and T.V. Shahbazyan, Surf. Sci. Rep. 40, 1 (2000).
${ }^{12}$ A.P. Heberle, J.J. Baumberg, and K. Köhler, Phys. Rev. Lett. 75, 2598 (1995).

${ }^{13}$ X. Marie, P. Le Jeune, T. Amand, M. Brousseau, J. Barrau, and M. Paillard, Phys. Rev. Lett. 79, 3222 (1997).

${ }^{14}$ J. Fernandez-Rossier, C. Tejedor, and R. Merlin, Semicond. Sci. Technol. 15, R65-R80 (2000).

${ }^{15}$ P. Nozières and C.T. De Dominicis, Phys. Rev. 178, 1097 (1969).

${ }^{16}$ T. Uenoyama and L.J. Sham, Phys. Rev. Lett. 65, 1048 (1990).

${ }^{17}$ K. Ohtaka and Y. Tanabe, Rev. Mod. Phys. 62, 929 (1990).

${ }^{18}$ V.M. Axt and Stahl, Z. Phys. B: Condens. Matter 93, 195 (1994); Th. Östreich, K. Schönhammer, and L.J. Sham, Phys. Rev. B 58, 12920 (1998).

${ }^{19}$ G. Bastard, Wave Mechanics Applied to Semiconductor Heterostructures (Les Éditons the Physique, Paris, 1988).

${ }^{20}$ S. Mukamel, Principles of Nonlinear Optical Spectroscopy (Oxford University, Oxford, 1994).

${ }^{21}$ M.U. Wehner, M.H. Ulm, D.S. Chemla, and M. Wegener, Phys. Rev. Lett. 80, 1992 (1998).

${ }^{22} \tau<0$ means that the pulse labeled as 2 appearing twice in Eq. (12) arrives to the sample before than the pulse 1 appearing only once in Eq. (12).

${ }^{23}$ N.A. Fromer, C. Schüller, D.S. Chemla, T.V. Shahbazyan, I.E. Perakis, K. Maranowski, and A.C. Gossard, Phys. Rev. Lett. 83, 4646 (1999).

${ }^{24}$ G.F. Giuliani and J.J. Quinn, Phys. Rev. B 26, 4421 (1982).

${ }^{25}$ P.W. Anderson and G. Yuval, Phys. Rev. Lett. 23, 89 (1969).

${ }^{26}$ J. von Delft and H. Schoeller, Ann. Phys. (Leipzig) 7, 225 (1998). 